Real Analysis Exchange

Vol. 23(2), 1997-1998, pp. 653-669

L. Zajíček*, Department of Math. Anal., Charles University, Sokolovská 83, 18600 Prague 8, Czech Republic e-mail: zajicek@karlin.mff.cuni.cz

\title{
ORDINARY DERIVATIVES VIA SYMMETRIC DERIVATIVES AND A LIPSCHITZ CONDITION VIA A SYMMETRIC LIPSCHITZ CONDITION
}

\begin{abstract}
If a subset $A$ of the real line is a countable union of closed, strongly symmetrically porous sets, then there exists a Lipschitz everywhere symmetrically differentiable function $f$ such that $A$ is the set of all nondifferentiability points of $f$. Since there are closed strongly symmetrically porous sets of Hausdorff dimension 1, our construction answers a problem posed by J. Foran in 1977. We also obtain results concerning smallness of the set of points at which a continuous function fulfills the symmetric Lipschitz condition but does not fulfill the ordinary Lipschitz condition.
\end{abstract}

\section{Introduction and Notation}

In this article we will consider real functions defined on the real line $\mathbb{R}$. By the symmetric derivative of a function $f$ at a point $x \in \mathbb{R}$ we mean

$$
f_{s}^{\prime}(x):=\lim _{h \rightarrow 0+} \frac{f(x+h)-f(x-h)}{2 h} ;
$$

we consider here only finite symmetric derivatives.

Let us recall that $f$ satisfies the Lipschitz condition at $x \in \mathbb{R}$ if

$$
\limsup _{h \rightarrow 0}\left|\frac{f(x+h)-f(x)}{h}\right|<\infty .
$$

Key Words: symmetric derivative, symmetric Lipschitz property, symmetric porosity Mathematical Reviews subject classification: 26A24, 28A05

Received by the editors December 8, 1997

*The research was supported by the grants GAČR 201/94/0474 and GAČR 201/97/1161 
Following [9], we say that a function $f: \mathbb{R} \rightarrow \mathbb{R}$ fulfills the symmetric Lipschitz condition at a point $x$ if

$$
\limsup _{h \rightarrow 0+}\left|\frac{f(x+h)-f(x-h)}{2 h}\right|<\infty .
$$

We shall use the following notation.

$$
\begin{aligned}
C(f) & =\{x: f \text { is continuous at } x\}, \\
D(f) & =\left\{x: f^{\prime}(x) \in \mathbb{R} \text { exists }\right\}, \\
S D(f) & =\left\{x: f_{s}^{\prime}(x) \in \mathbb{R} \text { exists }\right\}, \\
L(f) & =\{x: f \text { fulfils the Lipschitz condition at } x\}
\end{aligned}
$$

and

$$
S L(f)=\{x: f \text { fulfils the symmetric Lipschitz condition at } x\} .
$$

Let $E \subset \mathbb{R}, x \in \mathbb{R}$ and $r>0$. Then we define $s(E, x, r)$ as the supremum of all numbers $h>0$ for which there exists a $p>0$ such that $p+h \leq$ $r,(x+p, x+p+h) \cap E=\emptyset$ and $(x-p-h, x-p) \cap E=\emptyset$. The symmetric porosity of $E$ at $x$ is defined as

$$
p^{s}(E, x):=\limsup _{r \rightarrow 0+} \frac{s(E, x, r)}{r} .
$$

We say that $E$ is symmetrically porous at $x$ ( $d$-symmetrically porous at $x$ ) if $p^{s}(E, x)>0\left(p^{s}(E, x) \geq d\right)$. If $E$ is 1 -symmetrically porous at $x$, we say that $E$ is strongly symmetrically porous at $x$.

A set $E \subset \mathbb{R}$ is symmetrically porous (strongly symmetrically porous, $d$ symmetrically porous) if it is symmetrically porous (strongly symmetrically porous, $d$-symmetrically porous) at each of its points.

A set $E$ is called $\sigma$-symmetrically porous ( $\sigma$-strongly symmetrically porous, $\sigma-d$-symmetrically porous) if it is a countable union of symmetrically porous (strongly symmetrically porous, $d$-symmetrically porous) sets.

Khintchine [5] proved that the set $S D(f) \backslash D(f)$ is of Lebesgue measure zero for each measurable function $f$. Foran [4] (and independently also Ponomarev [7]) constructed a continuous function on $\mathbb{R}$ which has a finite symmetric derivative everywhere and is differentiable at no point of a nonempty perfect set. Thus the set $S D(f) \backslash D(f)$ can be uncountable also for a continuous function $f$. Foran in his article asked two questions. 
The first question asks whether there exists a continuous function $f$ which has a finite symmetric derivative everywhere and the set of all non-differentiability points of $f$ has a positive Hausdorff dimension. Note that Foran observed that this set has Hausdorff dimension zero in his example. Thomson ([9], p. 266) conjectured that this question has positive answer; we will see that his intuition was right on target.

Foran's second question, which asks whether each perfect set of measure zero is the set of all non-differentiability points for a continuous function which has a finite symmetric derivative everywhere, was answered negatively by Belna, Evans and Humke [1]. They proved that, for a continuous function $f$, the set $S D(f) \backslash D(f)$ is $\sigma$-porous and used the fact $([10])$ that there exists a perfect set of measure zero which is not $\sigma$-porous.

Evans in [2] factually proved the following result which improves the result of [1] and generalizes the previous result of (the preprint of) [12].

Theorem E. Let $f: \mathbb{R} \rightarrow \mathbb{R}$ be given. Then the set $(S D(f) \backslash D(f)) \cap \overline{C(f)}$ is $\sigma-(1-\varepsilon)$-symmetrically porous for each $0<\varepsilon<1$.

This result was formulated in [2] in the case $S D(f) \subset \overline{C(f)}$ only, but it is obvious that the same arguments give also the above result.

In [12] this result was proved for continuous $f$ only. The fact that Theorem $\mathrm{E}$ is a true improvement of the result of [1] was proved in [3].

The natural problem of a complete characterization (or at least a complete characterization of smallness) of sets $S D(f) \backslash D(f)$ for continuous $f$ (or for symmetrically differentiable continuous $f$ is Problem 42 of [9]) and seems to be open.

The main result (Theorem 3.2) of the present article says that if $A \subset \mathbb{R}$ is a countable union of closed strongly symmetrically porous sets, then $A=$ $S D(f) \backslash D(f)$ for a Lipschitz everywhere symmetrically differentiable function $f$. The corresponding construction is similar to that of [7] but it contains also some small new ideas.

Theorem E and Theorem 3.2 suggest that, if a simple characterization discussed above exists, it must probably deal with a type of symmetric porosity. We obtain a simple characterization in the class of perfect symmetric sets only. However, this result is strong enough to easily imply a positive answer to Foran's first question mentioned above. The set $\mathbb{R} \backslash D(f)$ can have Hausdorff dimension 1 for a Lipschitz everywhere symmetrically differentiable function $f$.

In Section 4 we consider the size of $S L(f) \backslash L(f)$. First we show (Theorem 4.1) that the notes [12] and [2] easily give that $(S L(f) \backslash L(f)) \cap \overline{C(f)}$ is $\sigma$ strongly symmetrically porous for each $f: \mathbb{R} \rightarrow \mathbb{R}$. Thus $S L(f) \backslash L(f)$ is 
$\sigma$-strongly symmetrically porous if $\overline{C(f)}=\mathbb{R}$, in particular for each Baire one function $f$.

The basic constructions used in the proof of Theorem 3.2 easily give that, if $F \subset \mathbb{R}$ is a countable union of closed strongly symmetrically porous sets, then there exists a continuous function $f$ such that $F \subset S L(f) \backslash L(f)$ (even $F \subset S D(f) \backslash L(f))$. Note that we cannot demand here $S L(f)=\mathbb{R}$, see Remark 4.9 .

The same constructions give a complete characterization of those symmetric, perfect sets that are of the form $S L(f) \backslash L(f)$ (or $S D(f) \backslash L(f)$ ) for a continuous function $f$. In particular, we obtain that $S D(f) \backslash L(f)$ can be of Hausdorff dimension 1 for a continuous function $f$.

It should be mentioned that Theorem 4.1 was originally contained in an unpublished note written (and originally also submitted for publication) in 1996. The results of Section 3 were presented on the Workshop in Real Analysis, Budapest 21.6.-24.6.1997.

We adopt the following notation.

The four Dini derivates of $f$ at $x$ are denoted by $D^{+} f(x), D_{+} f(x), D^{-} f(x)$ and $D_{-} f(x)$.

Lebesgue measure on $\mathbb{R}$ is denoted by $\lambda$.

If $I \subset \mathbb{R}$ is an interval, we frequently write $|I|$ instead of $\lambda I$.

The symbols $\bar{A}$ and $\operatorname{int} A$ denote the closure and the interior of a set $A$, respectively. The distance of two sets $A, B$ is denoted by $\operatorname{dist}(A, B)$.

We say that a function $f$ is $K$-Lipschitz if $f$ is Lipschitz with the constant $K$.

The support of $f$ is $\operatorname{supp}(f):=\overline{\{x \in \mathbb{R}: f(x) \neq 0\}}$.

\section{Lemmas and Basic Constructions}

We start with the following useful technical definitions.

\section{Definition 2.1.}

(a) By an $I$-system $\mathcal{I}$ we mean a finite (possibly empty) disjoint system of nonempty bounded closed intervals. We put

$$
\nu(\mathcal{I})=\sup \{|I|: I \in \mathcal{I}\} .
$$

(b) Let $\mathcal{I}, \mathcal{K}$ be $I$-systems and let $c>0$. We say that $\mathcal{I}$ is $c$-embedded in $\mathcal{K}$ if

(b1) for each $I \in \mathcal{I}$ there exists $K \in \mathcal{K}$ such that $I \subset K$ and $\operatorname{dist}(I, \mathbb{R} \backslash \bigcup \mathcal{K})=\operatorname{dist}(I, \mathbb{R} \backslash K)>c|I|$, and 


$$
\text { (b2) } \operatorname{dist}(I, J)>c|I| \text { whenever } I, J \in \mathcal{I}, I \neq J .
$$

The fact that a closed set is strongly symmetrically porous can be expressed in different ways. One of them uses the notion of $c$-embedding of $I$-systems; in the following lemma we formulate and prove the only implication we need.

Lemma 2.2. Let $F \subset \mathbb{R}$ be a nonempty bounded closed strongly symmetrically porous set and let $\left(c_{n}\right)_{n=1}^{\infty}$ be a sequence such that $c_{n}>1$ and $c_{n} \rightarrow \infty$. Then there exist I-systems $\left(\mathcal{I}_{n}\right)_{n=0}^{\infty}$ such that, for every $n \in \mathbb{N}$,

(i) $\mathcal{I}_{n}$ is $c_{n}$-embedded in $\mathcal{I}_{n-1}$,

(ii) $\nu\left(\mathcal{I}_{n}\right)<1 / c_{n}$ and

(iii) $F=\bigcap_{k=0}^{\infty} \bigcup \mathcal{I}_{k}$.

Proof. Find $a, b \in \mathbb{R}$ such that $F \subset(a, b)$ and put $\mathcal{I}_{0}=\{[a, b]\}$. Further suppose that $k \in \mathbb{N}$ and that $\mathcal{I}_{0}, \ldots, \mathcal{I}_{k-1}$ were constructed so that, for every $0 \leq n \leq k-1$, the following conditions hold:

(a) conditions (i) and (ii) hold whenever $n>0$,

(b) $F \subset \operatorname{int}\left(\bigcup \mathcal{I}_{n}\right)$ and

(c) $F \cap I \neq \emptyset$ whenever $I \in \mathcal{I}_{n}$.

We want to construct $\mathcal{I}_{k}$ such that (i), (ii), (b) and (c) hold for $n=k$. Since $F \subset \operatorname{int}\left(\bigcup \mathcal{I}_{k-1}\right)$, we have $\rho:=\operatorname{dist}\left(F, \mathbb{R} \backslash \bigcup \mathcal{I}_{k-1}\right)>0$. Since $F$ is strongly symmetrically porous, we can assign numbers $p_{x}>0, h_{x}>0$ to every $x \in F$ so that

$$
\begin{aligned}
\left(x+p_{x}, x+p_{x}+h_{x}\right) \cap F & =\emptyset,\left(x-p_{x}-h_{x}, x-p_{x}\right) \cap F=\emptyset, \\
h_{x} & >8 c_{k} p_{x} \text { and } \\
6 p_{x} c_{k} & <\min (1, \rho) .
\end{aligned}
$$

By the Borel covering lemma, we can find points $x_{1}, \ldots, x_{m} \in F$ such that, putting $p_{i}:=p_{x_{i}}, h_{i}:=h_{x_{i}}$, the intervals $\left(x_{i}-p_{i}-h_{i}, x_{i}+p_{i}+h_{i}\right), i=1, \ldots, m$, cover the set $F$. By (1) we also have that the system of intervals $\Phi:=\left\{J_{i}:=\right.$ $\left.\left[x_{i}-p_{i}, x_{i}+p_{i}\right]: i=1, \ldots, m\right\}$ covers $F$. Moreover, we may and will suppose that

$$
\text { no proper subsystem of } \Phi \text { covers } F \text {. }
$$

Now put

$$
\mathcal{I}_{k}=\left\{\left[x_{i}-2 p_{i}, x_{i}+2 p_{i}\right]: i=1, \ldots, m\right\} .
$$


Let $1 \leq i, j \leq m$ and $y_{i} \in\left[x_{i}-2 p_{i}, x_{i}+2 p_{i}\right], y_{j} \in\left[x_{j}-2 p_{j}, x_{j}+2 p_{j}\right]$. We may and will suppose $p_{j} \leq p_{i}$. First we shall show that $y_{i} \neq y_{j}$. In fact, otherwise clearly

$$
\left[x_{j}-p_{j}, x_{j}+p_{j}\right] \subset\left(x_{i}-5 p_{i}, x_{i}+5 p_{i}\right)
$$

and therefore (2) implies

$$
\left[x_{j}-p_{j}, x_{j}+p_{j}\right] \subset\left(x_{i}-p_{i}-h_{i}, x_{i}+p_{i}+h_{i}\right) .
$$

Consequently (1) gives $\left[x_{j}-p_{j}, x_{j}+p_{j}\right] \cap F \subset\left[x_{i}-p_{i}, x_{i}+p_{i}\right]$ which contradicts (4).

Thus we know that $\mathcal{I}_{k}$ is an $I$-system. Further (1) implies $\left|x_{i}-x_{j}\right| \geq p_{i}+h_{i}$. Consequently, using (2), we have

$$
\begin{aligned}
\left|y_{i}-y_{j}\right| & \geq p_{i}+h_{i}-4 p_{i}>8 c_{k} p_{i}-3 p_{i}>5 c_{k} p_{i}>c_{k} \lambda\left[x_{i}-2 p_{i}, x_{i}+2 p_{i}\right] \\
& \geq c_{k} \lambda\left[x_{j}-2 p_{j}, x_{j}+2 p_{j}\right] .
\end{aligned}
$$

If, moreover, $z \in \mathbb{R} \backslash \bigcup \mathcal{I}_{k-1}$ is given, then (3) gives $\left|z-x_{i}\right| \geq \rho>6 p_{i} c_{k}$. Therefore

$$
\left|z-y_{i}\right| \geq 6 p_{i} c_{k}-2 p_{i}>4 p_{i} c_{k} \geq c_{k} \lambda\left[x_{i}-2 p_{i}, x_{i}+2 p_{i}\right] .
$$

Thus we have shown that (i) holds for $n=k$. By (3) we obtain $\lambda\left[x_{i}-2 p_{i}, x_{i}+\right.$ $\left.2 p_{i}\right]=4 p_{i}<4 / 6 c_{k}<1 / c_{k}$ which implies that (ii) holds for $n=k$ as well.

The validity of (b) and (c) for $n=k$ is obvious. Thus the sequence $\left(\mathcal{I}_{n}\right)_{n=0}^{\infty}$ is well defined. It clearly satisfies (i) and (ii); (iii) follows by (b),(c),(ii) and the assumption $c_{n} \rightarrow \infty$.

In the following construction, we build more complicated functions from basic building blocks; functions $g_{I}$ which are assigned to each closed bounded interval $I$. We need only the following properties of $g_{I}$.

(a) $g_{I}$ is 4 -Lipschitz and of the class $C^{1}$ on $\mathbb{R}$.

(b) $\operatorname{supp}\left(g_{I}\right) \subset I$ and $g_{I}(x) \geq 0$ for each $x \in \mathbb{R}$.

(c) $g_{I}$ attains its maximum which equals $|I|$ at the center $c$ of $I$ and $g_{I}(c+h)=$ $g_{I}(c-h)$ for all $h \in \mathbb{R}$.

It is easy to see that such functions exist.

The following construction depends on a parameter $0 \leq \alpha<1$; we shall apply it in the following with $\alpha=0$ and $\alpha=1 / 2$.

Construction Let $0 \leq \alpha<1$ and $d>1$ be given. Further let $I$-systems $\mathcal{I}$ and $\mathcal{K}$ such that $\mathcal{I}$ is $4 d^{2}$-embedded in $\mathcal{K}$ be given We shall construct a function $\varphi=\varphi(\alpha, d, \mathcal{I})$ (which does not depend on $\mathcal{K}$ ) in the following way. 
To every interval $I=[a, b] \in \mathcal{I}$, we assign the "right" interval $I^{r}:=$ $[b+d|I|, b+2 d|I|]$ and the "left" interval $I^{l}:=[a-2 d|I|, a-d|I|]$. Put

$$
\varphi=\varphi(\alpha, d, \mathcal{I}):=\sum_{I \in \mathcal{I}} d^{\alpha}\left(g_{I^{r}}+g_{I^{l}}\right) .
$$

We shall need the properties of $\varphi$ which are proved in the following lemma.

Lemma 2.3. The function $\varphi=\varphi(\alpha, d, \mathcal{I})$ constructed above has the following properties:

(P1) $\varphi$ is a non-negative $C^{1}$ function on $\mathbb{R}$ with a compact support.

(P2) $|\varphi(x)| \leq d^{\alpha+1} \nu(\mathcal{I})$ for each $x \in \mathbb{R}$.

(P3) $\operatorname{dist}(\operatorname{supp}(\varphi), \bigcup \mathcal{I})>0$ and $\operatorname{supp} \varphi \subset \bigcup \mathcal{K}$.

(P4) $\varphi$ is 4-Lipschitz in the case $\alpha=0$.

(P5) If $x \in \bigcup \mathcal{I}$ and $h>0$, then $|\varphi(x+h)-\varphi(x-h)| / 2 h \leq 4 d^{\alpha-1}$.

(P6) For every $x \in \bigcup \mathcal{I}$ there exists $0<h<3 \nu(\mathcal{I}) d$ such that $\varphi(x+h) / h>$ $d^{\alpha} / 3$.

Proof. To each $I \in \mathcal{I}$ assign an "enlarged" interval $I^{*}:=\left[a-2 d^{2}|I|, b+\right.$ $\left.2 d^{2}|I|\right]$. Observe that

$$
I^{r} \cup I^{l} \subset I^{*} \text { and }\left\{I^{*}: I \in \mathcal{I}\right\} \text { is a disjoint system. }
$$

The first claim of (5) is obvious. To prove the second one, suppose on the contrary that $I^{*} \cap J^{*} \neq \emptyset$ for different $I, J$ from $\mathcal{I}$. We may and will suppose $|I| \geq|J|$. Then the distance between $I$ and $J$ is clearly at most $2 d^{2}|I|+$ $2 d^{2}|J| \leq 4 d^{2}|I|$ which contradicts the assumption that $\mathcal{I}$ is $4 d^{2}$-embedded in $\mathcal{K}$.

Using (5) and the definitions of $\varphi$ and $g_{I}$ we immediately obtain the properties (P1)-(P4).

To prove (P5), suppose that $x \in I=[a, b] \in \mathcal{I}$ and $h>0$ are given. Denote $c:=(a+b) / 2$. If $0<h \leq d|I|$, then clearly $\varphi(x+h)=\varphi(x-h)=0$. If $d|I|<h \leq 2 d^{2}|I|$, then the points $c+h, c-h, x+h, x-h$ belong to $I^{*}$ and (5) implies that $\varphi=g_{I^{r}}+g_{I^{l}}$ on $I^{*}$. Thus $\varphi(c+h)-\varphi(c-h)=0$ and

$$
\begin{aligned}
|\varphi(x+h)-\varphi(x-h)| \leq & |\varphi(c+h)-\varphi(c-h)|+|\varphi(c+h)-\varphi(x+h)| \\
& +|\varphi(c-h)-\varphi(x-h)| \\
\leq & 0+4 d^{\alpha}|c-x|+4 d^{\alpha}|c-x| \leq 8 d^{\alpha}|I|<8 d^{\alpha-1} h .
\end{aligned}
$$


If $h>2 d^{2}|I|$, then (5) gives that either $\varphi(x+h)=g_{J^{l}}(x+h)$ or $\varphi(x+h)=$ $g_{J^{r}}(x+h)$ for an interval $J \in \mathcal{I}, J \neq I$. In both cases $|\varphi(x+h)| \leq d^{1+\alpha}|J|$. If $\varphi(x+h) \neq 0$, then clearly $h+2 d|I| \geq \operatorname{dist}(I, J) \geq 4 d^{2}|J|$. Consequently $h>2 d^{2}|J|$ and thus we have

$$
\left|\frac{\varphi(x+h)}{2 h}\right| \leq \frac{d^{\alpha+1}|J|}{4 d^{2}|J|}=\frac{d^{\alpha-1}}{4} .
$$

Similarly we obtain $|\varphi(x-h) / 2 h| \leq d^{\alpha-1} / 4$. The inequalities proved above immediately give (P5).

To prove (P6), suppose that an $x \in I \in \mathcal{I}$ is given. Put $h:=b+\frac{3}{2} d|I|-x$. Then clearly $0<h<3 d|I|<3 \nu(\mathcal{I}) d$ and

$$
\frac{\varphi(x+h)}{h}=\frac{d^{\alpha+1}|I|}{h}>\frac{d^{\alpha+1}|I|}{3 d|I|}=\frac{d^{\alpha}}{3} .
$$

Lemma 2.4. Suppose that $0 \leq \alpha<1,(\mathcal{I})_{n=0}^{\infty}$ and $\left(d_{n}\right)_{n=1}^{\infty}$ are given so that all $\mathcal{I}_{n}$ are $I$-systems, $d_{n}>1$. In addition assume

(i) $\mathcal{I}_{n}$ is $4 d_{n}^{2}$-embedded in $\mathcal{I}_{n-1}$ for every $n \in \mathbb{N}$,

(ii) $\left(d_{n}\right)^{\alpha+1} \nu\left(\mathcal{I}_{n}\right) \rightarrow 0,\left(d_{n}\right)^{\alpha+1} \nu\left(\mathcal{I}_{n}\right) \leq 1$ for every $n \in \mathbb{N}$ and

(iii) $\sum_{n=1}^{\infty}\left(d_{n}\right)^{\alpha-1}<\infty$.

Denote $F:=\bigcap_{n=0}^{\infty} \cup \mathcal{I}_{n}$. Then there exists a function $f=f_{\alpha}$ such that

(iv) $f$ is continuous, $|f(x)| \leq 1$ for every $x \in \mathbb{R}$ and $f$ is 4 -Lipschitz in the case $\alpha=0$,

(v) $f$ is a $C^{1}$ function on $\mathbb{R} \backslash F$,

(vi) $f_{s}^{\prime}(x)=0$ for every $x \in F$ and

(vii) if $x \in F$, then

$$
\begin{aligned}
& \text { (a) } D^{-} f(x) \leq 0 \\
& \text { (b) } D^{+} f(x) \geq 1 / 3 \text { in the case } \alpha=0 \text { and } \\
& \text { (c) } D^{+} f(x)=\infty \text { in the case } \alpha>0 .
\end{aligned}
$$

Proof. Let $\varphi_{n}=\varphi\left(\alpha, d_{n}, \mathcal{I}_{n}\right)$ be the functions from the Construction. Put $f=f_{\alpha}=\sum_{n=1}^{\infty} \varphi_{n}$. By (i) and (P3) of Lemma 2.3 we have that the supports of the functions $\varphi_{n}$ are pairwise disjoint. This fact, (P1), (P2), (P4) and (ii) 
easily imply (iv) and (v). To prove (vi) suppose that $x \in F$ and $\varepsilon>0$ are given. Observe that (P3) implies that $\varphi_{k}^{\prime}(x)=0$ for each $k$. Using also (P5) and (iii) we obtain

$$
\begin{aligned}
\limsup _{h \rightarrow 0}\left|\frac{f(x+h)-f(x-h)}{2 h}\right| \leq & \limsup _{h \rightarrow 0} \sum_{k=1}^{n}\left|\frac{\varphi_{k}(x+h)-\varphi_{k}(x-h)}{2 h}\right| \\
& +\limsup _{h \rightarrow 0} \sum_{k=n+1}^{\infty}\left|\frac{\varphi_{k}(x+h)-\varphi_{k}(x-h)}{2 h}\right| \\
\leq & +\sum_{k=n+1}^{\infty} 4 d^{\alpha-1}<\varepsilon,
\end{aligned}
$$

if $n$ is chosen sufficiently large. Thus $f_{s}^{\prime}(x)=0$.

If $x \in F$, then $f(x)=0$, and since $f$ is non-negative, we obtain (vii),(a).

For each index $n$ by (P6) we can find an $h_{n}$ such that $0<h_{n}<3 \nu\left(\mathcal{I}_{n}\right) d_{n}$ and $\varphi\left(x+h_{n}\right) / h_{n}>\left(d_{n}\right)^{\alpha} / 3$. Since $d_{n}>1$, we obtain by (ii) that $h_{n} \rightarrow 0$. Since

$$
\frac{f\left(x+h_{n}\right)-f(x)}{h_{n}} \geq \frac{\varphi_{n}\left(x+h_{n}\right)}{h_{n}}>\frac{\left(d_{n}\right)^{\alpha}}{3}
$$

and $d_{n} \rightarrow \infty$ by (iii), we obtain (vii),(b) and (vii),(c).

\section{$3 \quad$ Symmetric Derivatives}

Proposition 3.1. Let $F \subset \mathbb{R}$ be a bounded closed strongly symmetrically porous set. Then there exists a non-negative 1-Lipschitz function $g$ such that $|g(x)| \leq 1$ for every $x \in \mathbb{R}, g$ is a $C^{1}$-function on $\mathbb{R} \backslash F$ and, for every $x \in F$, we have

$$
g(x)=0, g_{s}^{\prime}(x)=0, D^{-} g(x) \leq 0 \quad \text { and } \quad D^{+} g(x) \geq 1 / 12 .
$$

Proof. Put $d_{n}:=2 n^{2}$ and apply Lemma 2.2 to $F$ and $c_{n}:=4\left(d_{n}\right)^{2}$. The resulting $I$-systems $\left(\mathcal{I}_{n}\right)_{n=0}^{\infty}$ clearly satisfy assumptions (i)-(iii) of Lemma 2.4 for $\alpha=0$. Now it is clearly sufficient to find the corresponding $f=f_{0}$ and put $g:=f / 4$.

Theorem 3.2. Let $A \subset \mathbb{R}$ can be written in the form $A=\bigcup_{n=1}^{\infty} F_{n}$, where each $F_{n}$ is closed and strongly symmetrically porous. Then there exists a Lipschitz symmetrically differentiable function $f$ on $\mathbb{R}$ such that $A$ is the set of all non-differentiability points of $f$. 
Proof. We may suppose that all $F_{n}$ are bounded. For each $n$, we apply Proposition 3.1 to $F=F_{n}$ and obtain a corresponding function $g=g_{n}$. Now put $f:=\sum_{n=1}^{\infty}(26)^{-n} g_{n}$. Obviously, $f$ is a Lipschitz function.

Let $x \in \mathbb{R}$ be given and put $D:=\sum_{n=1}^{\infty}(26)^{-n}\left(g_{n}\right)_{s}^{\prime}(x)$. For each $\varepsilon>0$ find $k \in \mathbb{N}$ such that $\sum_{n=k+1}^{\infty}(26)^{-n}<\varepsilon / 3$ and $h_{0}>0$ such that

$$
\left|\sum_{n=1}^{k}(26)^{-n} \frac{g_{n}(x+h)-g_{n}(x-h)}{2 h}-\sum_{n=1}^{k}(26)^{-n}\left(g_{n}\right)_{s}^{\prime}(x)\right|<\frac{\varepsilon}{3}
$$

for every $0<h<h_{0}$. Since each $g_{n}$ is 1 -Lipschitz, we conclude that

$$
\begin{aligned}
& \left|\frac{f(x+h)-f(x-h)}{2 h}-D\right| \\
= & \left|\sum_{n=1}^{\infty}(26)^{-n} \frac{g_{n}(x+h)-g_{n}(x-h)}{2 h}-\sum_{n=1}^{\infty}(26)^{-n}\left(g_{n}\right)_{s}^{\prime}(x)\right| \\
\leq & \left|\sum_{n=1}^{k}(26)^{-n} \frac{g_{n}(x+h)-g_{n}(x-h)}{2 h}-\sum_{n=1}^{k}(26)^{-n}\left(g_{n}\right)_{s}^{\prime}(x)\right| \\
& +\left|\sum_{k+1}^{\infty}(26)^{-n} \frac{g_{n}(x+h)-g_{n}(x-h)}{2 h}\right|+\left|\sum_{k+1}^{\infty}(26)^{-n}\left(g_{n}\right)_{s}^{\prime}(x)\right| \\
< & \frac{\varepsilon}{3}+\frac{\varepsilon}{3}+\frac{\varepsilon}{3}=\varepsilon
\end{aligned}
$$

if $0<h<h_{0}$. Therefore $f_{s}^{\prime}(x)=D$ and thus $f$ is symmetrically differentiable.

Quite similar argument gives that $f^{\prime}(x)=\sum_{n=1}^{\infty}(26)^{-n}\left(g_{n}\right)^{\prime}(x)$ for each $x \in \mathbb{R} \backslash A$.

Let now a point $x \in A$ be fixed. Find $k \in \mathbb{N}$ such that $x \in F_{k}$ and $x \notin F_{n}$ for every $n<k$. Then the function $\sum_{n<k}(26)^{-n} g_{n}$ is differentiable at $x$ and

$$
D^{+}\left((26)^{-k} g_{k}\right)(x)-D^{-}\left((26)^{-k} g_{k}\right)(x) \geq 26^{-k} \frac{1}{12} \text {. }
$$

Since the function $\sum_{n=k+1}^{\infty}(26)^{-n} g_{n}$ is Lipschitz with the Lipschitz constant $\sum_{n=k+1}^{\infty}(26)^{-n}=(26)^{-k} / 25$, we conclude that

$$
D^{+} f(x)-D^{-} f(x) \geq \frac{1}{12}(26)^{-k}-\frac{2}{25}(26)^{-k}>0 .
$$

As an almost immediate consequence of this theorem and results of [3], we obtain the following result on symmetric perfect sets. We use here the notation 
from [6]. Namely, if a sequence $\lambda=\left(\lambda_{n}\right)_{n=1}^{\infty}$ with $0<\lambda_{n}<\frac{1}{2}$ is given, then we consider the symmetric perfect set (the "generalized Cantor set" in [6]) $C(\lambda) \subset$ $[0,1]$ which is constructed like the classical Cantor ternary set is so that, after the $n$-th step of construction, we obtain $2^{n}$ closed "remaining" intervals with the same length $\lambda_{1} \ldots \lambda_{n}$. Symmetric perfect sets are sometimes called also "symmetric Cantor sets" and/or determined by a sequence $\left(\alpha_{n}\right)_{n=1}^{\infty}, 0<\alpha_{n}<$ 1 (see [3]). Note that for $\alpha_{n}=1-2 \lambda_{n}$ the set $C\left(\alpha_{n}\right)$ from [3] coincides with the set $C(\lambda)$ from $[6]$.

Proposition 3.3. Let $C=C(\lambda) \subset[0,1]$ be a symmetric perfect set. Then the following statements are equivalent.

(i) $\liminf \lambda_{n}=0$.

(ii) There exists a Lipschitz function $f$ on $\mathbb{R}$ which has a finite symmetric derivative at all points, is of the class $C^{1}$ outside $C$ but $f^{\prime}(x)$ exists at no point $x \in C$.

(iii) There exists a function $f$ on $\mathbb{R}$ such that $C \subset(S D(f) \backslash D(f)) \cap \overline{C(f)}$.

Proof. Theorem 3 and Theorem 5 of [3] give that (i) holds iff $C$ is strongly symmetrically porous. Thus Proposition 3.1 immediately gives the implication $(i) \Rightarrow(i i)$. The implication $(i i) \Rightarrow(i i i)$ is trivial. To prove the implication (iii) $\Rightarrow($ i) suppose that (i) fails. Then we know by Theorem 3 of [3] that there exists $\varepsilon>0$ such that

$$
C \text { is }(1-\varepsilon)-\text { symmetrically porous at no point of } C \text {. }
$$

By Theorem E (see Introduction) $C=\bigcup_{n=1}^{\infty} A_{n}$ where every $A_{n}$ is $(1-\varepsilon)$-symmetrically porous. By the Baire theorem we obtain that some $A_{n}$ is dense in a portion of $C$, which clearly contradicts (6).

The condition (i) implies that the Lebesgue measure of $C$ is zero but it is well-known that it implies no stronger smallness in the (Hausdorff) measure sense. In particular, there exists a symmetric perfect set $C$ of Hausdorff dimension 1 for which (i) holds. Thus Foran's first question (see Introduction) has a negative answer.

We shall now formulate and prove a more precise statement which deals with Hausdorff measures $\Lambda_{h}$ determined by non-decreasing functions $h:[0, \infty) \rightarrow[0, \infty), h(0)=0$ (see $[6]$ or $[8])$.

Proposition 3.4. Let $h: \mathbb{R} \rightarrow \mathbb{R}$ be an increasing function such that $h(0)=0$ and $h^{\prime}(0)=\infty$. Then there exists a symmetric perfect set $C$ and a Lipschitz function $f$ on $\mathbb{R}$ with the following properties. 
(i) $\Lambda_{h}(C)=\infty$, where $\Lambda_{h}$ is the Hausdorff measure determined by $h$.

(ii) The function $f$ is of the class $C^{1}$ outside $C$, has a finite symmetric derivative at all points and $f^{\prime}(x)$ exists at no point $x \in C$.

Proof. We will need the following fact (see [6], 4.11).

Fact Let $C=C(\lambda)$ be a symmetric perfect set. Put $s_{k}=\lambda_{1} \cdots \lambda_{k}$. If $g:[0, \infty) \rightarrow[0, \infty)$ is a continuous increasing function such that $g\left(s_{k}\right)=2^{-k}$, then $1 / 4 \leq \Lambda_{g}(C(\lambda)) \leq 1$.

For each natural number $k$ choose $\delta_{k}>0$ such that

$$
\frac{h(x)}{x}>(k+2) \text { ! whenever } 0<x \leq \delta_{k} .
$$

Further choose an increasing sequence of natural numbers $\left(n_{k}\right)_{k=1}^{\infty}$ such that $n_{1}>2$ and $2^{-n_{k}}<\delta_{k}$. Let $\left(p_{n}\right)_{n=1}^{\infty}$ be any fixed sequence such that

$$
0<p_{n}<1 \text { and } p:=\prod_{1}^{\infty} p_{n}>0 .
$$

Now put $\lambda_{n}=1 / k$ if $n=n_{k}$ and $\lambda_{n}=p_{n} / 2$ if no such $k$ exists. Clearly there exists a continuous increasing function $h^{*}:[0, \infty) \rightarrow[0, \infty)$ such that $h^{*}(0)=0$ and $h^{*}\left(\lambda_{1} \cdots \lambda_{n}\right)=2^{-n}$. By the above mentioned fact we have

$$
1 / 4 \leq \Lambda_{h^{*}}(C(\lambda)) \leq 1
$$

To prove $\Lambda_{h}(C(\lambda))=\infty$, by Theorem 40 of [8] it is suffices to establish that $\lim _{x \rightarrow 0+} \frac{h^{*}(x)}{h(x)}=0$. To this end, consider $0<x \leq \lambda_{1} \ldots \lambda_{n_{1}+1}$ and the corresponding index $n=n(x)$ for which $\lambda_{1} \cdots \lambda_{n+1}<x \leq \lambda_{1} \cdots \lambda_{n}$. Since clearly $n>n_{1}$, there exists the unique index $k=k(x)$ such that $n_{k} \leq n<n_{k+1}$. Since $\lambda_{1} \cdots \lambda_{n+1} \leq 2^{-n_{k}}<\delta_{k}$, we obtain

$$
\begin{aligned}
\frac{h^{*}(x)}{h(x)} & \leq \frac{2^{-n}}{h\left(\lambda_{1} \cdots \lambda_{n+1}\right)} \leq \frac{2^{-n}}{(k+1) ! \cdot \lambda_{1} \cdots \lambda_{n+1}} \\
& \leq \frac{2^{-n} \cdot(k+1) !}{(k+2) ! \cdot p 2^{-(n+1)}}=\frac{2}{p(k+2)} .
\end{aligned}
$$

Since clearly $k(x) \rightarrow \infty$ when $x \rightarrow 0+$, we are done.

\section{A Symmetric Lipschitz Condition}

In the first part of this section we show how the notes [12] and [2] give the following theorem. 
Theorem 4.1. For each function $f: \mathbb{R} \rightarrow \mathbb{R}$, the set $(S L(f) \backslash L(f)) \cap \overline{C(f)}$ is $\sigma$-strongly symmetrically porous.

This theorem immediately implies, for example, the following result.

Proposition 4.2. Let $f: \mathbb{R} \rightarrow \mathbb{R}$ be a function of Baire class one. Then the set of all points at which $f$ fulfills the symmetric Lipschitz condition but does not fulfill the Lipschitz condition is $\sigma$-strongly symmetrically porous.

Note that the above theorem is analogous to [11, Theorem 2] which asserts that, for each function $f: \mathbb{R} \rightarrow \mathbb{R}$, the set of all points at which $f$ fulfills an one-sided Lipschitz condition but does not fulfill the Lipschitz condition is $\sigma$-strongly porous.

M. J. Evans in [2, Proposition 1] proved the following result.

Proposition 4.3. For each function $f: \mathbb{R} \rightarrow \mathbb{R}$, the set $(S L(f) \cap \overline{C(f)}) \backslash C(f)$ is $\sigma$-strongly symmetrically porous.

Thus to prove our Theorem 4.1 it is sufficient to prove that

$$
(S L(f) \backslash L(f)) \cap C(f) \text { is } \sigma \text {-strongly symmetrically porous. }
$$

We will show that (7) easily follows from the following Lemma 4.4 which is essentially the main part of [12, Lemma 1].

Lemma 4.4. Let $f: \mathbb{R} \rightarrow \mathbb{R}$ be a function, $B>0$ and $1>\varepsilon>0$. For a natural number $m$ denote by $S_{m}$ the set of all points $x \in \mathbb{R}$ at which

$D^{+} f(x)>B$ and

$$
\frac{f(x+h)-f(x-h)}{2 h}<\frac{\varepsilon B}{8} \text { whenever } 0<h<\frac{1}{m} .
$$

Then $S_{m} \cap C(f)$ is $(1-\varepsilon)$-symmetrically porous.

It is necessary to note that in the proof of $[12$, Lemma 1$]$ it is only proved that $S_{m}$ is $(1-\varepsilon)$-symmetrically porous for a continuous function $f$. However, as was pointed out and used in [2], the assumption of global continuity of $f$ is not used in the proof and thus the conclusion of the above lemma holds.

To prove (7), suppose that a point $x \in M:=(S L(f) \backslash L(f)) \cap C(f)$ is given. Then we can clearly find a natural number $m$ such that

$$
\left|\frac{f(x+h)-f(x-h)}{2 h}\right|<m \quad \text { whenever } \quad 0<h<\frac{1}{m} .
$$

Thus, denoting by $M_{m}$ the set of all $x \in M$ for which (9) holds, we see that $M=\bigcup_{m=1}^{\infty} M_{m}$ and that it is sufficient to prove that each $M_{m}$ is $\sigma$-strongly 
symmetrically porous. Since $x \in L(f)$ clearly iff all four Dini derivates of $f$ at $x$ are finite, we have

$$
\begin{aligned}
M_{m}= & \left(M_{m} \cap\left\{x: D^{+} f(x)=\infty\right\}\right) \cup\left(M_{m} \cap\left\{x: D_{+} f(x)=-\infty\right\}\right) \\
& \cup\left(M_{m} \cap\left\{x: D^{-} f(x)=\infty\right\}\right) \cup\left(M_{m} \cap\left\{x: D_{-} f(x)=-\infty\right\}\right) .
\end{aligned}
$$

Considering the functions $f(-x),-f(x)$ and $-f(-x)$ we easily see that it is sufficient to prove that the set

$$
Z_{m}:=M_{m} \cap\left\{x: D^{+} f(x)=\infty\right\}
$$

is strongly symmetrically porous. To this end choose an arbitrary $1>\varepsilon>0$ and find $B>0$ such that $\varepsilon B / 8>m$. Then (9) and consequently also (8) is satisfied for each $x \in Z_{m}$. Since also $D^{+} f(x)=\infty>B$ for each $x \in Z_{m}$, our Lemma 4.4 implies that $Z_{m}$ is $(1-\varepsilon)$-symmetrically porous. Thus $Z_{m}$ is 1 -symmetrically porous, i.e. it is strongly symmetrically porous.

The second part of this section, which concerns the sets $S L(f) \backslash L(f)$ is analogical to Section 3 which deals with the sets $S D(f) \backslash D(f)$.

Proposition 4.5. Let $F \subset \mathbb{R}$ be a bounded, closed, strongly symmetrically porous set. Then there exists a non-negative continuous function $f$ such that $|f(x)| \leq 1$ for every $x \in \mathbb{R}, f$ is a $C^{1}$ function on $\mathbb{R} \backslash F$ and, for every $x \in F$, we have $f(x)=0, f_{s}^{\prime}(x)=0$ and $D^{+} f(x)=\infty$. In particular, $S D(f)=S L(f)=\mathbb{R}$ and $F=\mathbb{R} \backslash L(f)$.

Proof. Put $d_{n}:=2 n^{3}$ and apply Lemma 2.2 to $F$ and $c_{n}:=4\left(d_{n}\right)^{2}$. The resulting $I$-systems $\left(\mathcal{I}_{n}\right)_{n=0}^{\infty}$ obviously satisfy the assumptions (i)-(iii) of Lemma 2.4 for $\alpha=1 / 2$. Then the function $f$ from the assertion of Lemma 2.4 has clearly all required properties.

Now we can simply prove an analogy of Proposition 3.3 on symmetric perfect sets.

Proposition 4.6. Let $C=C(\lambda)$ be a symmetric perfect set. Then the following statements are equivalent.

(i) $\liminf \lambda_{n}=0$.

(ii) There exists a continuous symmetrically differentiable function $f$ which is $C^{1}$ on $\mathbb{R} \backslash C$ and $D^{+} f(x)=\infty$ for each $x \in C$.

(iii) There exists a function $f$ on $\mathbb{R}$ such that $C \subset(S L(f) \backslash L(f)) \cap \overline{C(f)}$. 
Proof. If (i) holds then $C$ is strongly symmetrically porous by Theorem 5 of [3] and thus Proposition 4.5 implies (ii). The implication $(i i) \Rightarrow(i i i)$ is trivial. The implication $($ iii $) \Rightarrow(i)$ can be easily proved, using Theorem 4.1 and Theorem 3 of [3] and imitating the proof of the implication $(i i i) \Rightarrow(i)$ of Proposition 3.3.

Quite similarly as in Proposition 3.4, we easily see that Proposition 4.6 implies that (for a continuous $f$ ) the Lebesgue null set $S D(f) \backslash L(f)$ (and thus also $S L(f) \backslash L(f))$ need not be small in any reasonable stronger (Hausdorff) measure sense.

Proposition 4.7. Let $h:[0, \infty) \rightarrow[0, \infty)$ be an increasing continuous function with $h(0)=0$ and $h_{+}^{\prime}(0)=\infty$. Then there exist a symmetric perfect set $C$ and a continuous symmetrically differentiable function $f$ such that $\Lambda_{h}(C)=\infty, \quad f$ is $C^{1}$ on $\mathbb{R} \backslash C$ and $C=\mathbb{R} \backslash L(f)$.

Theorem 4.8. Let $A \subset \mathbb{R}$ be written in the form $A=\bigcup_{n=1}^{\infty} F_{n}$, where each $F_{n}$ is closed and strongly symmetrically porous. Then there exists a continuous function $g$ on $\mathbb{R}$ such that, for every $x \in A, g_{s}^{\prime}(x) \in \mathbb{R}$ exists but $g$ is not Lipschitz at $x$; in particular $A \subset S L(g) \backslash L(g)$.

Proof. We may suppose that each set $F_{n}$ is bounded. For each $n$, let $f=f_{n}$ be a function which corresponds to $F=F_{n}$ by Proposition 4.5 . It is easy to see that, for each $n \in \mathbb{N}$, there exists a closed (even discrete) set $D_{n} \subset \mathbb{R}$ such that $D_{n} \cap A=\emptyset$ and the distance function

$$
d_{n}(x):=\operatorname{dist}\left(x, F_{1} \cup \cdots \cup F_{n} \cup D_{n}\right)
$$

is bounded by 1 . Now put

$$
g_{1}:=f_{1}, g_{n}:=n^{-2} f_{n}\left(d_{n-1}\right)^{2} \text { for } n>1 \text { and } g:=\sum_{n=1}^{\infty} g_{n}
$$

The function $g$ is clearly continuous on $\mathbb{R}$.

Now let $x \in A$ be given and let $k$ be a natural number with $x \in F_{k}$ and $x \notin F_{n}$ for each $n<k$. Observe that each $d_{n}$ has clearly finite both onesided derivatives, and therefore a finite symmetric derivative, at any point $y \notin F_{1} \cup \cdots \cup F_{n} \cup D_{n}$. The same property is satisfied also for functions $\left(d_{n}\right)^{2}$, which are clearly also bounded by 1 and Lipschitz on $\mathbb{R}$. By the above observation, the function $\sum_{n<k} g_{n}$ is Lipschitz on $\mathbb{R}$ and has a finite symmetric derivative at $x$.

Now denote $s:=\sum_{n=k+1}^{\infty} g_{n}$. For every $n \geq k+1$, clearly $g_{n}(x)=$ $d_{n-1}(x)=0$ and $\left|g_{n}(x+h)\right| \leq n^{-2}\left(d_{n-1}(x+h)\right)^{2} \leq n^{-2} h^{2}$ for every $h \in \mathbb{R}$. 
Consequently, for every $h \neq 0$,

$$
\left|\frac{s(x+h)-s(x)}{h}\right| \leq|h|^{-1} \sum_{n=k+1}^{\infty} n^{-2} h^{2}=|h| \sum_{n=k+1}^{\infty} n^{-2} .
$$

Thus $s^{\prime}(x)=0$. Since both $f_{k}$ and $\left(d_{k-1}\right)^{2}$ have a finite symmetric derivative at $x$, we conclude that $g_{k}$ and $g$ have finite symmetric derivatives at $x$.

On the other hand, $g_{k}$ is not Lipschitz at $x$. In fact, suppose that $g_{k}$ is Lipschitz at $x$. Then, since we have observed that the function $\left(d_{k-1}\right)^{2}$ is Lipschitz at $x$ and $d_{k-1}(x) \neq 0$, we easily conclude that also $f_{k}=k^{2}\left(d_{k-1}\right)^{-2} g_{k}$ is Lipschitz at $x$, a contradiction. Since both $\sum_{n<k} g_{n}$ and $s$ are Lipschitz at $x$, we obtain that $g$ is not Lipschitz at $x$.

Remark 4.9. If $A$ in Theorem 4.8 is not nowhere dense, no corresponding function $g$ is symmetrically Lipschitz at all points. In fact, suppose that $f$ is a continuous function on $\mathbb{R}$ and $S L(f)=\mathbb{R}$. Put

$$
S_{n}:=\left\{x \in \mathbb{R}: \frac{|f(x+h)-f(x-h)|}{2 h} \leq n \text { whenever } 0<h<\frac{1}{n}\right\} .
$$

Then clearly $\mathbb{R}=\bigcup_{n=1}^{\infty} S_{n}$ and the continuity of $f$ easily implies that all $S_{n}$ are closed. Thus the Baire category theorem easily gives that each interval $I$ contains a subinterval $J$ which is contained in an $S_{m}$; it easily implies that $f$ is Lipschitz on $J$. Therefore $\mathbb{R} \backslash L(f)$ is nowhere dense.

\section{References}

[1] C. L. Belna, M. J. Evans, P. D. Humke, Symmetric and ordinary differentiation, Proc. Amer. Math. Soc. 72 (1978), 261-267.

[2] M. J. Evans, A note on symmetric and ordinary differentiation, Real Anal. Exchange 17 (1991-9-2), 820-826.

[3] M. J. Evans, P. D. Humke and K. Saxe, A symmetric porosity conjecture of Zajíček, Real Analysis Exch. 17 (1991-92), 258-271.

[4] J. Foran, The symmetric and ordinary derivative, Real Analysis Exch. 2 (1977), 105-108.

[5] A. Khintchine, Recherches sur la structure des fonctions measurable, Fund. Math. 9 (1927), 212-279.

[6] P. Matilla, Geometry of sets and measures in Euclidean spaces, Cambridge University Press 1995. 
[7] S. P. Ponomarev, Symmetric differentiable functions with a perfect set of non-differentiability points, (in Russian), Matem. Zametki 38 (1985), 30-38.

[8] C. A. Rogers, Haudorff measures, Cambridge University Press 1970.

[9] B. S. Thomson, Symmetric properties of real functions, Monographs and textbooks in pure and applied mathematics; 183, Marcel Dekker Inc., New York 1994.

[10] L. Zajíček, Sets of $(\sigma)$-porosity and sets of $(\sigma)$-porosity (q), Časopis Pěst. Mat. 101 (1976), 350-359.

[11] L. Zajíček, On the symmetry of Dini derivates of arbitrary functions, Comment. Math. Univ. Carolinae 22 (1981), 195-209.

[12] L. Zajičck, A note on the symmetric and ordinary derivative, Atti Sem. Mat. Fis. Univ. Modena XLI (1993), 263-267. 